\title{
Afrontamiento y tolerancia al estrés académico en situación de confinamiento según Calixta Roy
}

\section{Addressing and tolerance to academic stress in confinement situation according to Calixta Roy}

\author{
Diana Acosta-Salazar (iD) ${ }^{1}$, Patricia Lapeira-Panneflex (iD) 2 , Edna Osorio-Duran (iD) 3 \\ 1. Universidad del Magdalena. Santa Marta, Colombia. Correo: dianis276@hotmail.com - https://orcid.org/0000-0003-4409-9893 \\ 2. Universidad del Magdalena. Santa Marta, Colombia. Correo: patrilape0618@hotmail.com - https://orcid.org/0000-0002-1088-1345 \\ 3. Universidad del Magdalena. Santa Marta, Colombia. Correo: emosoriod@hotmail.com - https://orcid.org/0000-0003-4566-3118
}

Tipología: Artículo de investigación científica y tecnológica

Para citar este artículo: Acosta-Salazar D, Lapeira-Panneflex $\mathrm{P}$, Osorio-Duran E Afrontamiento y tolerancia al estrés académico en situación de confinamiento según Calixta Roy. Duazary. 2021 septiembre; 18(3 número especial): 26-35. Doi: https://doi.org/10.21676/2389783X.4260

Palabras

clave: afrontamiento; adaptación; enfermería.

\section{RESUMEN}

Estudio cualitativo, fenomenológico, cuyo objetivo fue describir la adaptación al estrés académico con base en el Modelo de la Adaptación de Roy de los estudiantes del área básica con asignaturas en repitencia del Programa de Enfermería de una universidad pública. La muestra fueron 12 estudiantes, como criterio de inclusión estar matriculados cursando una asignatura del área básica por segunda vez y participación voluntaria. Técnica de recolección de la información fue entrevista semiestructurada. Los resultados evidenciaron el uso de los cuatro modos de adaptación definidos por Roy, en el modo de adaptación fisiológico presentaron trastornos de sueño y preocupación; en el modo autoconcepto establecen mecanismos de ayuda frente a su dificultad; en el modo función de rol realizan función de estudiante, hijo; en el de interdependencia por iniciativa los estudiantes afianzaron sus creencias para obtener fortalecimiento. Se concluye que los estudiantes en repitencia de una asignatura del área básica afrontaron el estrés académico adaptándose según el Modelo descrito por Calixta Roy, emplean los cuatro modos de adaptación, y en cuanto a los factores descrito por Roy los más utilizados por los estudiantes fueron el factor 4: Procesamiento sistemático, y el factor 5: Conociendo y relacionando.

\section{ABSTRACT}

Keywords: Coping; Adaptation; Nursing.
Qualitative, phenomenological study, the objective of which was to describe the adaptation to academic stress based on the Roy Adaptation Model for students in the basic area with repetitive subjects in the Nursing Program of a public university. The sample was 12 students, the inclusion criteria who were enrolled taking a subject in the basic area for the second time and voluntary participation. Information gathering technique was semi-structured interview. The results obtained were that the students used the four adaptation modes defined by Roy in the physiological one presented sleep disorders and worry; in the self-concept mode, they establish help mechanisms for their difficulty; in role role mode they perform student, child role; In the interdependence initiative, students strengthened their beliefs to obtain strength. The factors described by Roy the most used are factor 4: Systematic processing, and factor 5: Knowing and relating. It is concluded that the students in repetition of a subject in the basic area face academic stress adapting according to the Model described by Calixta Roy, using the four adaptation modes: physiological, self-concept, role function and interdependence, in terms of the factors described by Roy's most used by students were factor 4: Systematic processing, and factor 5 : Knowing and relating. 


\section{INTRODUCCIÓN}

La situación de confinamiento es un escenario con gran impacto en el bienestar emocional, físico y psicológico. La interrupción de manera brusca de la cotidianeidad del diario vivir; asistir a la universidad, ir al trabajo, el encierro de todas las personas durante un tiempo largo, se ha constituido en factores causantes de estrés. En el tiempo de confinamiento, se han establecido dos elementos que aquejan el bienestar físico y psicológico, y estos son, la privación de costumbres y rutinas; y el estrés psicosocial, según estudio realizado en China sobre los efectos de la pandemia por COVID-19 ${ }^{1}$.

Los contextos que hacen parte de una pandemia circunscriben múltiples fuentes de estrés. Las investigaciones realizadas sobre ambientes $y$ entornos estresantes asienten que los principales aspectos relacionados con el impacto psicológico son el temor a infectarse con el virus, expresión de sentimientos de fracaso y tedio, incapacidad para suplir requerimientos básicos y no tener conocimiento y patrones de acción definidos ${ }^{2}$.

Sobre la base de las consideraciones anteriores frente a situaciones de estrés, en el ser humano según Flórez et $a l^{3}$ la capacidad de afrontamiento y adaptación le permite responder mediante comportamientos, pensamientos o emociones a eventos estresantes.

Para Pérez $z^{4}$ en una persona, las estrategias de afrontamiento hacen referencia a los esfuerzos, mediante conductas que son manifestadas o se interiorizan, para hacer frente a las demandas internas y ambientales, y los conflictos entre ellas. Así mismo, para Casullo ; el afrontamiento se puede definir como "las estrategias conductuales y cognitivas para lograr una transición y una adaptación efectiva".

La presente investigación, es producto del interés por el estudio del tema de afrontamiento $y$ adaptación según el modelo de Callista Roy para evaluar la adaptación al estrés que presentan los estudiantes de enfermería del área básica en una asignatura en repitencia durante el primer semestre de 2020, cuando por la situación sanitaria originada por la COVID-19 fue decretado el confinamiento obligatorio en nuestro país, el 11 de marzo de 2020, luego de la declaración de pandemia por la Organización Mundial de la Salud ${ }^{6}$, lo cual forzó a los estudiantes a jornadas académicas a través de las pantallas en una modalidad enteramente virtual, presentándose reacciones desmotivadoras durante su proceso de formación ${ }^{7}$. Por su parte Lunley y Provenzano ${ }^{8}$ describen que el estrés consigue afectar el desarrollo académico de un estudiante, ocasionando una interrupción en contra de su voluntad que perturba su actuación de cara a sus compromisos académicos, tales como, instruirse, concentrarse y ser aplicado.

El proceso de afrontamiento y adaptación, Roy ${ }^{9}$ los redefine como los patrones innatos y adquiridos de las formas de manejar y responder a los cambios del ambiente en las situaciones de la vida diaria y los periodos críticos, mediante comportamientos directamente enfocados a alcanzar el dominio, la supervivencia, el crecimiento y la trascendencia.

Los procesos de afrontamiento según Roy y Prangtip ${ }^{10}$ están dados por el subsistema regulador $y$ el cognitivo. Por lo que el primero es innato, responde a través de unos canales neuronales, químicos y endocrinos. Los estímulos ambientales actúan como entradas al sistema nervioso y afectan el equilibrio hidroelectrolítico, el equilibrio ácido básico y el sistema endocrino. De esta manera las respuestas son automáticas e inconscientes, y se expresan fundamentalmente en el modo fisiológico. El segundo, permite el enfrentamiento de los estímulos en ese proceso de pensar, sentir y actuar; para de esta manera interpretar esos estímulos tanto interno como externos.

Roy ${ }^{11}$ reconoce que el afrontamiento constituye una variable crucial para comprender el efecto del estrés sobre la salud y la enfermedad; para lo cual, el afrontamiento es clave en el manejo de la enfermedad, el mantenimiento de la salud o bien la recuperación. Los seres humanos a través del tiempo son seres adaptables, esto se logra por medio de procesos de aprendizaje; por tal motivo el 
estudiante universitario está en constante cambio de tal forma que la percepción que tiene de los contextos a las cuales se enfrenta son individuales y desiguales para cada uno ${ }^{12}$.

La presente investigación, es producto del interés por el estudio del tema de afrontamiento $y$ adaptación según el modelo de Callista Roy. Para ello, se propuso como objetivo principal describir la adaptación al estrés académico en situación de confinamiento con base en el Modelo de la Adaptación de Roy de los estudiantes del área básica con asignaturas en repitencia del Programa de Enfermería de una universidad pública de la ciudad de Santa Marta durante el primer semestre de 2020.

\section{MATERIALES Y METODOS}

\section{Diseño}

Esta Investigación es de tipo cualitativo y fenomenológico, ya que intenta captar lo subjetivo como un medio de comprender e interpretar las experiencias personales ${ }^{13}$ permitió acercarnos a la realidad vivenciada por los estudiantes y cómo afrontan las dificultades, carencias para adaptarse a la situación académica que enfrentan. La fenomenología se fundamenta en el estudio de las experiencias de vida, respecto de un suceso, desde la perspectiva del sujeto ${ }^{14}$

\section{Participantes de la investigación}

La población está compuesta por estudiantes de la Universidad del Magdalena, una universidad pública departamental cuya sede principal está ubicada en la ciudad de Santa Marta - Colombia. Los participantes en esta investigación fueron 12 estudiantes del área básica del programa de enfermería con asignaturas en repitencia o cursándola por segunda vez quienes accedieron voluntariamente participar en el estudio, 6 de la asignatura de Morfofisiología I y 6 de la asignatura de Morfofisiología II, cursando tercero y cuarto semestre del programa de Enfermería de la Universidad del Magdalena respectivamente, con el fin de evaluar la adaptación al estrés que presentan los estudiantes de enfermería del área básica en una asignatura en repitencia durante el primer semestre de 2020,

El criterio de inclusión fue que estuvieran matriculados, cursando una asignatura del área básica por segunda vez y que accedieran a participar voluntariamente. No se rechazó ningún participante. El único criterio de exclusión fue no estar cursando la asignatura de Morfofisiología I o Morfofisiología II por segunda vez, en semestre del programa de Enfermería de la Universidad del Magdalena.

\section{Técnica de recolección de la información}

La entrevista semiestructurada fue la técnica de recolección de información escogida para este estudio, ya que permite contar con una guía de entrevista elaborada con base en la literatura consultada respecto al tema y el objetivo del estudio y permite flexibilidad de aclarar las dudas por el entrevistador ${ }^{15}$.

\section{Procedimiento}

Se invitó a participar a todos los estudiantes del área básica que estuvieran cursando por según vez una asignatura, esta convocatoria se realizó a través de los correos electrónicos registrados en el módulo de admisiones de la plataforma de la Universidad del Magdalena, indicando el objetivo de la investigación y el respeto por la confidencialidad. Dado el confinamiento obligatorio debido a la situación sanitaria por la COVID-19, se programa cita vía WhatsApp, y se realiza llamada telefónica explicando el objetivo de la investigación y posteriormente se envía por correo electrónico el consentimiento informado. Finalmente se efectúa entrevista vía telefónica, solicitando el permiso para grabar la entrevista. Se procede a transcribir la información y los datos fueron examinados cualitativamente, a través del análisis de contenido de los relatos de los estudiantes, según los modos de adaptación en la esfera psicosocial del Modelo de la Adaptación de Roy.

El análisis de los resultados se realizó teniendo en cuenta los 4 modos de adaptación establecidos por Roy (fisiológico, auto concepto, función del rol e interdependencia) y los factores de la estrategia de 
afrontamiento (recursivo y centrado, reacciones físicas y enfocadas, procesos de alerta, proceso sistemático y conocer y relacionar).

\section{Declaración sobre los aspectos éticos}

El presente estudio adoptó las normas establecidas en la Resolución $8430^{16}$ de 1993 del Ministerio de salud en Colombia para investigación en seres humanos. Considerándose sin riesgo ya que no se realizaron intervenciones que pusieran en riesgo a los estudiantes que participaron, se les comunicó previamente los objetivos de la investigación, se les aseguró la confidencialidad de la información, participación voluntaria y firma de consentimiento informado. El estudio fue aprobado por el Comité de Investigación de la Facultad de Ciencias de la salud de la Universidad del Magdalena, institución en donde se realizó la investigación.

\section{RESULTADOS}

\section{Caracterización de la información}

Los 12 estudiantes universitarios que participaron en la investigación son pertenecientes al área básica del Programa de Enfermería, 6 cursando la asignatura de Morfofisiología I y 6 cursando la asignatura de Morfofisiología II cursando tercer y cuarto semestre respectivamente, 8 de sexo biológico femenino y 4 de sexo biológico masculino, en edades entre los 18 a 21 años, solteros, residentes todos en la capital del departamento del Magdalena, pertenecientes a los estratos 1, 2 y 3.

En los modos de adaptación tenemos:

- Fisiológico: Horas de sueño, nivel de estrés y ansiedad: se identificaron altos niveles de ansiedad relacionados con la dinámica del proceso de aprendizaje por vía remota, lo cual desencadenó trastornos en el sueño, preocupación por el componente evaluativo de la asignatura dado el gran volumen de contenidos y poca concentración en el desarrollo de las temáticas, teniendo en cuenta que su experiencia anterior con la asignatura no fue la mejor.
- Autoconcepto: Cambio de conducta, acata órdenes, eficiencia bajo estrés, dominio de situaciones difíciles, desenvolvimiento: se establecieron patrones comportamentales que indicaron falencias en la realización de actividades de manera autónoma, los estudiantes se muestran muy dependientes de la supervisión del docente en todas las actividades relacionadas al desarrollo del material de estudio, las actividades que les demandan esfuerzo físico y mental se ven afectadas por niveles de ansiedad y estrés reflejados en poca concentración en las instrucciones dadas, a la vez establecen mecanismos de ayuda frente a su dificultad como la revisión de temas de manera adicional, asistencia a monitorias de temáticas especificas según su necesidad de aprendizaje y fortalecimiento de conceptos revisados anteriormente.

- Función del rol: Situación sanitaria de la COVID19, entorno familiar, sustento económico: los estudiantes reaccionaron de múltiples formas según las influencias del medio externo, cada individuo cumple un papel distinto en la sociedad, según su situación: madre, hijo, padre, estudiante. Lo cual llevo a modificaciones en su conducta, tales como, afectación en los hábitos de estudio dado que en el ambiente familiar actual debían realizar otras actividades simultaneas al estudio, el confinamiento limitaba el acceso a fuentes de información, consulta e incluso a las mismas actividades académicas; el factor económico y de sustento influyó de manera considerable en la adquisición de materiales que permitieran el uso de herramientas virtuales.

- Interdependencia: Expresa sus temores, se aísla o se relaciona con facilidad, búsqueda de apoyo espiritual: de manera dinámica y por iniciativa propia los estudiantes afianzaron sus creencias como forma de obtener fortalecimiento en medio de la situación sanitaria experimentada, que de una u otra forma les cambio la vida. La autoimagen y el dominio del papel social de cada individuo interacciona con las personas de su entorno, ejerciendo y recibiendo influencias. 
Esto crea relaciones de interdependencia, que pueden ser modificadas por los cambios del entorno.

Con relación a los cinco factores propuestos por Roy, los tres primeros miden los comportamientos, las reacciones y los recursos de afrontamiento que utilizan las personas, y los dos últimos miden las estrategias empleadas para sobrellevar la situación, con base en esto los resultados fueron:

Factor uno: recursivo y centrado. Este factor refleja comportamientos de la utilización del yo y de los recursos que se concentran para expandir las entradas del sistema cognitivo de la información, considerando actitudes respecto a la solución de problemas como el ser creativo y buscar resultados. Los estudiantes inicialmente se muestran renuentes al componente virtual dado la densidad de los contenidos, a la vez se involucran en el proceso de formación fortaleciendo en lo posible las herramientas requeridas para la adquisición del material.

Factor dos: reacciones físicas y enfocadas. Resaltan las reacciones físicas y la fase de entrada para el manejo de situaciones. El confinamiento y la no asistencia de manera presencial a las clases forja un componente de preocupación, generando en el estudiante fijación en la situación problema lo cual no le permite avanzar, haciéndose necesario implementar estrategias de dominio conductual que redireccionen las acciones en la realización de tareas para avanzar.

Factor tres: proceso de alerta. Representa los comportamientos del yo personal respecto a las características de cada persona como las expectativas, los valores, las virtudes y las metas, y los comportamientos del yo físico haciendo referencias a aspecto físicos, funcionalidad, salud y estado de enfermedad, compuesto por las sensaciones corporales y la imagen corporal. Los estudiantes establecen recursos que les permitan afrontar la situación, creación de grupos virtuales para revisión de contenidos, espacios de pausas activas, momentos de instructivos como estrategias de otros y/o experiencias para afrontar crisis; lo cual se constituye en una estrategia de dominio cognitivo.
Factor cuatro: procesamiento sistemático. Describe las estrategias personales y físicas para hacerse cargo de las situaciones y manejarlas metódicamente. La dinámica de las clases por vía remota demanda una organización en la utilización del tiempo y de recursos, los estudiantes experimentaron agotamiento debido al no mantenimiento y /o equilibrio entre el descanso y las actividades académicas de la asignatura, siendo necesario establecer agendas académicas de los contenidos a desarrollar.

Factor cinco: conocer y relacionar. Describe las estrategias que utiliza la persona para recurrir a sí misma y a otros usando la memoria y la imaginación. Los estudiantes identificaron de manera temprana y oportuna, cada una de las situaciones problemas que afectaban de una u otra forma su aprendizaje, intercambiaron experiencias significativas en el enfrentamiento del confinamiento, lo cual les permitió afianzar y fortalecer mecanismos propios según cada necesidad. Durante las jornadas se realizaban lúdicas que permitían el buen sentido del humor como terapia de relajación para el manejo de situaciones de difíciles. 


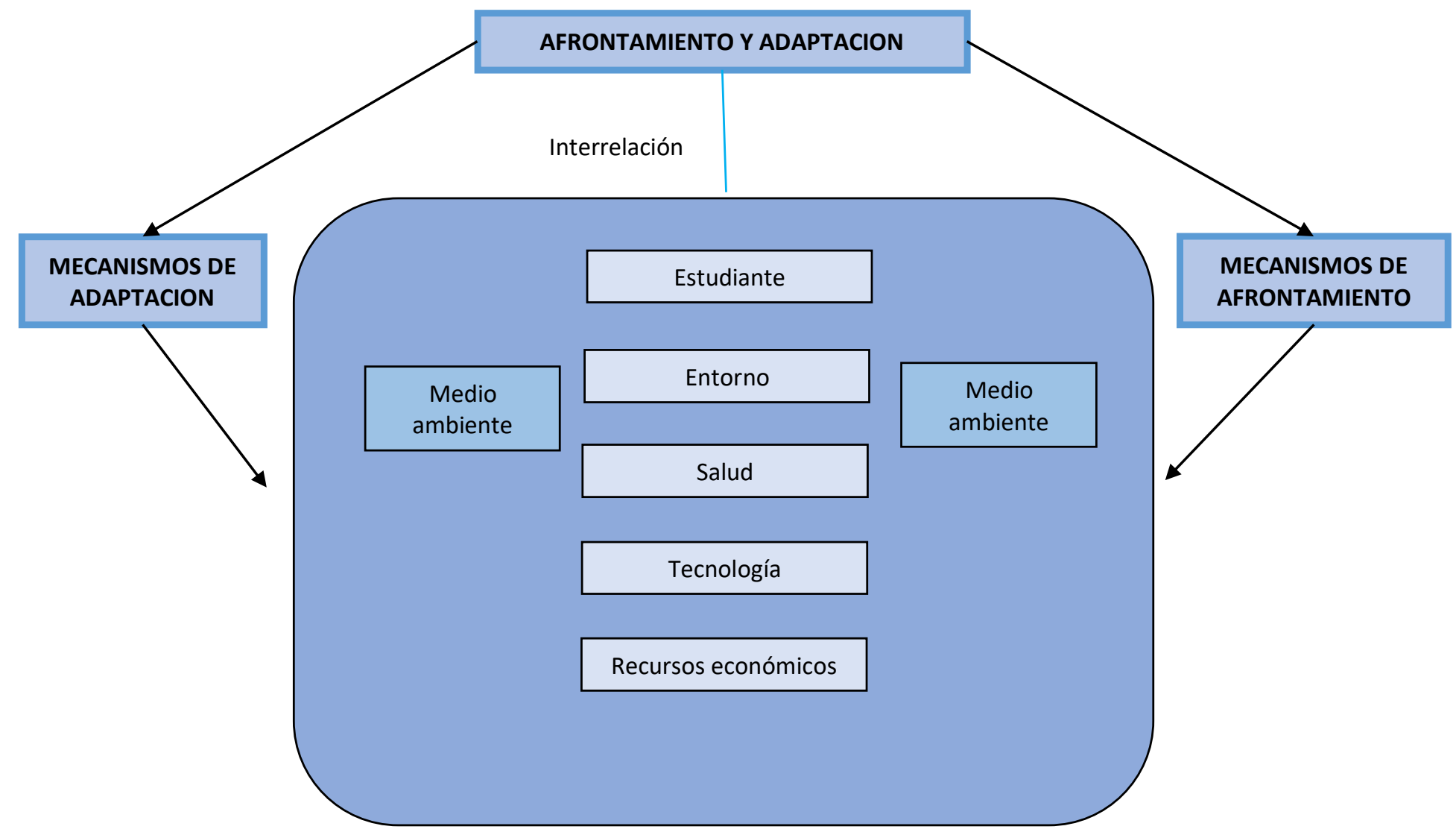

Focales: duración del confinamiento, virtualidad, clases remotas
NEGATIVAS: desesperanza, angustia, desmotivación
Residuales: emociones, sentimientos, creencias
POSITIVAS: autonomía, seguridad, esperanza 


\section{DISCUSIÓN}

Los seres humanos, como seres adaptativos, tienen la capacidad de crear cambios en el ambiente ${ }^{17}$, los recursos de afrontamiento innatos y adquiridos del ser humano busca ajustarse a un ambiente cambiante $^{18}$. Los estudiantes en repitencia de una asignatura del área básica, participantes en el presente estudio manifestaron cambios en los 4 modos de adaptación definidos por Roy, en el fisiológico presentaron trastornos del sueño, este síntoma también fue descrito en otra investigacion ${ }^{19}$ poca concentración y preocupación por el componente evaluativo.

En el modo de autoconcepto logran establecer mecanismos de ayuda frente a su dificultad como la revisión de temas de manera adicional, asistencia a monitorias de temáticas específicas. Al respecto Calixta Roy ${ }^{20}$, afirma que cuando la persona responde a los cambios del entorno de forma positiva, se favorece su integridad y su salud, si, por el contrario, la respuesta al cambio es ineficaz ocurren trastornos que llevan al fracaso.

El nivel de adaptación demuestra la interacción del estudiante con el ambiente, es decir, las modificaciones que ejecuta este para adaptarse a la nueva situación, mediante comportamientos directamente enfocados a alcanzar el dominio, la supervivencia, el crecimiento y la trascendencia. En cuanto al modo de función del rol el estudiante ante la nueva situación de confinamiento desempeñó diferentes roles dentro de su ambiente familiar como es el de hijo, algunos ayudar a el sustento económico de la familia, de acuerdo con el concepto dado por el modelo de Roy este es relacionado a la integración de la persona en la sociedad, así como a la buena relación con las personas que forman parte de su convivencia ${ }^{21}$.

En el modo de interdependencia el comportamiento de adaptación satisfactorio de los estudiantes se puede observar en la forma como de manera dinámica y por iniciativa propia los estudiantes afianzaron sus creencias como forma de obtener fortalecimiento en medio de la situación sanitaria experimentada. El manejo de estas estrategias de afrontamiento se relaciona con lo definido por Moos el cual alude que en el afrontamiento cognitivo se apela a la planeación, ensayo de una serie de acciones una serie de acciones a seguir, analizándose sus consecuencias, aceptando de esta forma la situación del evento estresante tratando de cambiarlo para obtener efectos positivos ${ }^{22}$. En lo referente a las estrategias de afrontamiento $y$ adaptación de los factores descritos por Roy el factor 4 Procesamiento sistemático, y el factor 5 Conociendo y relacionando fueron los más utilizados. Los estudiantes identificaron de manera temprana y oportuna, cada una de las situaciones problemas que afectaban de una u otra forma su aprendizaje, intercambiaron experiencias significativas en el enfrentamiento del confinamiento, lo cual les permitió afianzar y fortalecer mecanismos propios según cada necesidad, este hallazgo coincide con otra investigación ${ }^{23}$ lo cual confirma lo descrito por el modelo: cuando el individuo se enfrenta a un problema se edifican nuevos conocimientos a través de los recuerdos y las experiencias nuevas, lo cual le permite desarrollar nuevas capacidades o destrezas para la solución de problemas ${ }^{24}$.

Así mismo, Lozano, et $a l^{25}$ establece en su investigación, que el confinamiento ha tenido un gran impacto en los estudiantes universitarios primordialmente en el ámbito psicológico y académico, y lo fundamental de la relación de la satisfacción con la resiliencia.

Para Barraza ${ }^{26}$ las medidas de distanciamiento social, el confinamiento, el temor al contagio y la interrupción de actividades sociales, laborales y recreativas, han concebido niveles altos de estrés; exponiendo que es un mecanismo adaptativo de los seres humanos, compuesto por tres momentos: percepción, reacción y acción como respuesta.

Respecto a lo anterior, González ${ }^{27}$ manifiesta que la magnitud de la pandemia y lo que trajo consigo se podría considerar como una oportunidad que ayude a la reflexión sobre los mecanismos y estrategias en el proceso enseñanza - aprendizaje, con la finalidad que los estudiantes amplíen sus capacidades de autoaprendizaje. 
En lo mencionado a la capacidad de afrontamiento y tolerancia se establece un estilo de afrontamiento pasivo o activo en donde el estudiante se adapta a situaciones estresantes igual que los cuidadores de pacientes hospitalizados en Unidades de cuidados intensivos.

\section{CONSIDERACIONES FINALES}

Los estudiantes de enfermería repitentes de alguna asignatura del área básica lograron afrontar el estrés académico por la situación de confinamiento por la emergencia sanitaria originada por la COVID-19, adaptándose según el modelo descrito por Calixta Roy, lo cual produjo en el modo de adaptación fisiológica trastorno del sueño, preocupación por el componente evaluativo de la asignatura. En el modo de autoconcepto establecen mecanismos de ayuda frente a su dificultad como la revisión de temas de manera adicional, asistencia a monitorias de temáticas específicas. En lo referente a función de rol debieron realizar otras funciones además de ser estudiantes, rol de hijos y ayuda económica al hogar por la situación de confinamiento obligatorio. En el modo de interdependencia de manera dinámica y por iniciativa propia los estudiantes afianzaron sus creencias como forma de obtener fortalecimiento en medio de la situación sanitaria vivenciada.

En cuanto a las estrategias de afrontamiento y adaptación de los factores descritos por Roy el factor 4: Procesamiento sistemático, y el factor 5: Conociendo y relacionando fueron los más utilizados por los estudiantes entrevistados.

\section{DECLARACIÓN SOBRE CONFLICTOS DE INTERESES}

Los autores declaran que no existe conflicto de intereses

\section{CONTRIBUCIÓN DE LOS AUTORES}

Primer autor: concepción del estudio, realización de entrevistas, análisis e interpretación de los datos y redacción inicial.
Segundo autor: búsqueda, revisión de la literatura, revisión crítica del manuscrito, aprobación final de la versión que será publicada.

Tercer autor: revisión crítica del manuscrito, discusión, redacción final y aprobación final de la versión que será publicada.

\section{REFERENCIAS BIBLIOGRAFICAS}

1. Wang C, Pan R, Wan X, Tan Y, Xu L, Ho C, et al. Immediate Psychological Responses and Associated Factors during the Initial Stage of the 2019 Coronavirus Disease (COVID-19) Epidemic among the General Population in China. International Journal of Environmental Research and Public Health. 2020 marzo;17 (5):1729. Doi: https://doi.org/10.3390/ijerph17051729.

2. Brooks S, Webster R, Smith L, Woodland L, Wessely S, Greenberg N. The psychological impact of quarantine and how to reduce it: rapid review of the evidence. The lance. 2020 marzo;395(10227):912-920. Doi: https://doi.org/10.1016/S0140-6736(20)30460-8.

3. Flórez I, Herrera E, Carpio E, Veccino M, Zambrano $D$, Reyes $Y$, et al. Afrontamiento y adaptación en pacientes egresados de unidades de cuidados intensivos. Aquichan. 2011 abril; 11(1): 23-39. Doi: https://doi.org/10.5294/aqui.2011.11.1.2.

4. Pérez D. Nivel de uso de estrategias de afrontamiento por la familia ante el estrés, por la hospitalización de un pariente adulto en la Unidad de Cuidados Intensivos del HNGAI-2015. 2016. Lima: Lilipec;2017. Disponible: https://pesquisa.bvsalud.org/portal/resource/pt/bi blio-1114458?lang=es.

5. Zapata A, Bastida M, Quiroga A, Charra S, Leiva J. Evaluación del bienestar psicológico y estrategias de afrontamiento en padres con niños o adolescentes con retraso mental leve Psiencia. Latinoamericana de Ciencia Psicológica. 2013; 5(1):15-23. Disponible: https://www.redalyc.org/pdf/3331/333127392003. pdf. 
6. Organización Mundial de la Salud (OMS). Alocución de apertura del Director General de la OMS en la rueda de prensa sobre la COVID-19; 2020. Disponible en: https://www.who.int/es/directorgeneral/speeches/detail/who-director-general-sopening-remarks-at-the-media-briefing-on-covid19---11-march-2020.

7. Ruiz R, Navarro E. Estrés Académico En Los Estudiantes De Las Universidades Públicas De La Región Apurímac En Tiempos De Pandemia Covid19. Ciencia Latina. 2021 enero;5(1). Doi: http://dx.doi.org/10.37811/cl_rcm.v5i1.238.

8. Lumley M, Provenzano K. Stress management through written emotional disclosure improves academic performance among college students with physical symptoms. Journal of Educational Psychology. 2003;95(3):641-649. Doi: https://doi.org/10.1037/0022-0663.95.3.641.

9. Roy C. The Roy Adaptación model. Estados Unidos: Prentice Hall; 2008. Disponible en: https://www.iberlibro.com/Roy-Adaptation-Model3rd-Edition-Sister/11788711359/bd.

10. Molina - Chailan P, Huecha - Perez D. Estrategias de afrontamiento del Equipo de Enfermería de la Unidad de Cuidados Intensivos Med-Quirúrgicos Hospital Regional Concepción Posterremoto y Tsunami 27/02.Chilena de medicina intensiva. 2010; 25 (4): 193-198. Doi: https://www.imbiomed.com.mx/articulo.php?id=8 0294.

11. Roy C. El modelo de adaptación de Roy en el contexto de los modelos de enfermería, con ejemplos de aplicación y dificultades. Cultura de los cuidados. 2000 junio;7(8): 139:159. Doi: https://doi.org/10.14198/cuid.2000.7-8.17.

12. Facultad de Enfermería, G. de estudio. Análisis de los conceptos del modelo de adaptación de Callista Roy. Aquichan. 2000 abril;2(1). Disponible en:

https://aquichan.unisabana.edu.co/index.php/aqui chan/article/view/18.

13. Dyniewics AM. Metodologia da Pesquisa Em Saúde Para Iniciantes. Brasil: Difusão;2011.
14. Fuster D. Investigación cualitativa: Método fenomenológico hermenéutico. Propósitos y Representaciones. 2019;7(1):201-229. Doi: http://dx.doi.org/10.20511/pyr2019.v7n1.267.

15. Díaz-Bravo L, Torruco-García Uri, MartínezHernández $\mathrm{M}$, Varela-Ruiz $\mathrm{M}$, La entrevista, recurso flexible y dinámico. Investigación en Educación Médica. 2013;2(7):162-167. Disponible en: http://www.scielo.org.mx/scielo.php?pid=S200750572013000300009\&script=sci_arttext.

16. Colombia. Ministerio de salud y Protección Social. Resolución No 008430 de 1993 Por la cual se establecen las normas científicas, técnicas y administrativas para la investigación en salud. Universidad del Rosario. Disponible en: https://www.urosario.edu.co/Escuela-

Medicina/Investigacion/Documentos-deinteres/Files/resolucion_008430_1993.pdf.

17. Moreno - Fergusson ME. Aplicación del Modelo de Adaptación en un Servicio de Rehabilitación Ambulatoria. Aquichan. 2009 mayo;1(1). Disponible en:

https://aquichan.unisabana.edu.co/index.php/aqui chan/article/view/7/12.

18. Livneh H. Psychosocial Adaptation to Chronic Illness and Desability: A conceptual Framework. Bulletin. 2001;44(3):151-161. Disponible en: http://my.fit.edu/ tharrell/cbhc/Medical/Chronic\% 20IIIness\%20Adaptation/chronic\%20illness\%20ther apist\%20info.pdf.

19. Ahumada B, Henríquez C, Maureira F, Ruiz V. Estrés estudiantil un estudio desde la mirada cualitativa. Investigación Cualitativa. 2013 I 1, (15).Disponible en: http://www.ubiobio.cl/miweb/webfile/media/357/ Articulos/Art\%C3\%ADculo\%20Estr\%C3\%A9s\%20Estudiantil.pdf.

20. Islas P. Modelo de adaptación al sistema educativo universitario de los estudiantes del programa de enfermería. Internacional de Salud, Bienestar y Sociedad. 2015 julio;2(1).Disponible en: https://www.academia.edu/23186573/Modelo_de _adaptaci\%C3\%B3n_al_sistema_educativo_universi 
tario_de_los_estudiantes_del_programa_de_enfer mer\%C3\%Ada

21. Roy SC, Andrews HA. The Roy Adaptation Model: the definitive statement. Norwalk. Estados Unidos:Appleton e Lange;1991.

22. Jiménez - Ocampo V, Zapata - Gutiérrez L, Díaz - Suárez L. Capacidad de afrontamiento y adaptación de los familiares del paciente adulto hospitalizado en una unidad de cuidado intensivo. Aquichan. 2013 Julio;13(2):159-172. Disponible en: https://aquichan.unisabana.edu.co/index.php/aqui chan/article/view/2407/3220.

23. Veloza M, Moreno ME, Crespo O, Gutiérrez E. Proceso de afrontamiento y adaptación. Bogotá: El Manual Moderno; 2017.

24. Gutiérrez M. Adaptación y cuidado en el ser humano. Una visión de enfermería. Bogotá: El Manual Moderno; 2007.
25. Lozano-Díaz A, Fernández-Prados J, FigueredoCanosa V, Martínez- Martínez A. Impactos del confinamiento por el COVID-19 entre universitarios: Satisfacción Vital, Resiliencia y Capital Social Online. International Journal of Sociology of Education. 2020 http://doi.org/10.17583/rise.2020.5925.

26. Barraza A. El estrés de pandemia (COVID 19) en la población mexicana. Centro de Estudios Clínica e Investigación Psicoanalítica S.C, México; 2020. Disponible en http://www.upd.edu.mx/PDF/Libros/Coronavirus.p df.

27. González - Velázquez I. Estrés académico en estudiantes universitarios asociados a la pandemia por covid-19. Espacio i+d, innovación más desarrollo. 2020;9 (25). Doi: https://doi.org/10.31644/IMASD.25.2020.a10. 